CASE REPORT

\title{
Left ventricular haemangioma with papillary endothelial hyperplasia and liver involvement
}

\author{
C-D Kan, C-T Yae, Y-J Yang
}

Heart 2004;90:e49 (http://www.heartinl.com/cgi/content/full/90/8/e49). doi: 10.1136/hrt.2004.040154

\begin{abstract}
An intracardiac haemangioma with papillary endothelial hyperplasia (PEH) and liver involvement has not been previously reported in the English literature. This report describes a 65 year old man with a left ventricular haemangioma with PEH coexistent with multiple nodular hepatic haemangiomas. Transthoracic and transoesophageal echocardiography identified a large tumour in the left ventricular cavity with a pedicle connected to the apex. Abdominal sonography also identified multiple hyperechoic hepatic tumours. Magnetic resonance imaging showed hypervascularity of both the cardiac and hepatic lesions. The left ventricular tumour was totally resected and the liver nodules were biopsied. Tissue pathological study showed that both the left ventricular tumour and liver lesions were haemangiomas with PEH. The patient was discharged without complications postoperatively.
\end{abstract}

$\mathrm{P}$ apillary endothelial hyperplasia (PEH, Masson's pseudoangiosarcoma) is a vascular proliferative lesion that occurs as a reaction to traumatic vascular stasis rather than being a true neoplasm. ${ }^{1}$ The process is typically observed in the subcutaneous tissue of the finger, head, and neck, thyroid, and adrenal gland. It usually occurs in dilated vascular spaces or haemangiomas. ${ }^{12}$ Although most intracardiac haemangiomas with PEH are solitary, a few reports have described cardiac haemangiomas with PEH and the involvement of another organ. ${ }^{3}$ Herein, a rare case is described in a patient who was found to have a solitary mass in his heart and multiple nodules in his liver, which were ultimately diagnosed histopathologically as haemangiomas with PEH.

\section{CASE REPORT}

A 65 year old man was admitted after presenting with lower back pain and exertional dyspnoea that had developed over the preceding few weeks. The patient's history was unremarkable except for many years of heavy smoking. On admission, his blood pressure was 140/80 $\mathrm{mm} \mathrm{Hg}$ and his pulse was regular, with a rate of 60 beats/min. On auscultation, a grade II/VI systolic murmur was heard over the left sternal border and cardiac apex. A chest roentgenogram showed mild cardiomegaly, and a non-specific ECG showed normal sinus rhythm. Transthoracic and transoesophageal echocardiograms identified a cystic, focally calcified mass $2.7 \times 3.9 \mathrm{~cm}$ in size, located within a dilated left ventricular cavity. The mass appeared to float between the ventricle and mitral valve area, with a pedicle connecting it to the apex. Multiple hyperechoic hepatic nodules were also identified on a screening abdominal sonograph. The cardiac and hepatic lesions were found to be hypervascular on thoracic and abdominal magnetic resonance imaging (fig lA,B,C). Coronary angiograms showed normal coronary arteries with faint tumour staining within the left ventricle. An operation was performed through a median sternotomy, cardiopulmonary bypass, and cardioplegic arrest during which the left ventricular tumour was simultaneously approached through the aortic and mitral valves with the aid of a $5 \mathrm{~mm}$ endoscope. A pedunculated mass $(6 \times 3 \times 3 \mathrm{~cm})$, attached by its pedicle to the septum near the apex, was completely resected (fig 1D). The liver nodules were also biopsied during the rewarming stage. Macroscopically, the mass was well encapsulated and contained multiple lobules filled with blood. Some calcified spots were visible on the adjacent cardiac septum. The histopathological diagnosis was intravascular haemangioma with $\mathrm{PEH}$, characterised by papillary structures and branching vascular spaces, with an endothelium lining composed of flattened, innocent looking cells (fig 2A). Focal calcification was present in the subendocardium of the heart. The liver nodules had the same papillary structures within the liver parenchyma but had spared the portal areas (fig 2B). The postoperative clinical course was satisfactory except for slight liver function impairment noted biochemically.

\section{DISCUSSION}

PEH, a well recognised but infrequently reported histological diagnosis, is a common vascular tumour of soft tissues and was first described by Pierre Masson in 1923. ${ }^{2}$ The lesion is thought to be the result of reactive endothelial proliferation rather than neoplasia. It is characterised by papillary lobules of proliferating endothelial cells with an underlying fibrous stroma and often resembles angiosarcoma. ${ }^{25}$ It may occur in any blood vessel in the body but is commonly found on the fingers, head, neck, and trunk. PEH may occur either in a pure primary form, as a focal change in a pre-existing vascular lesion (haemangioma, pyogenic granuloma, or vascular malformation), and rarely in an extravascular location following organisation of a haematoma. ${ }^{56}$ It is generally accepted that stasis and thrombosis are prerequisites for the development of $\mathrm{PEH}^{2}$ A cardiac haemangioma with PEH was diagnosed in the present patient. Histologically, both the cardiac and hepatic lesions were associated with blood and thrombus, and contained multiple papillae with fibrous cores covered by a single layer of bland endothelial cells. The lesions were distinguishable from angiosarcoma by virtue of the more regular papillae and minimal endothelial cell mitosis. Cardiac haemangiomas constitute less than $2.8 \%$ of all cardiac masses. Case reports of solitary cardiac haemangiomas with $\mathrm{PEH}$ are rare; ${ }^{4}$ haemangiomas with PEH affecting both the heart and liver have not been previously described in the English literature. Because of the highly vascular nature of both the cardiac and hepatic lesions on magnetic resonance imaging, cardiac malignancy with liver metastases became the presumptive, 


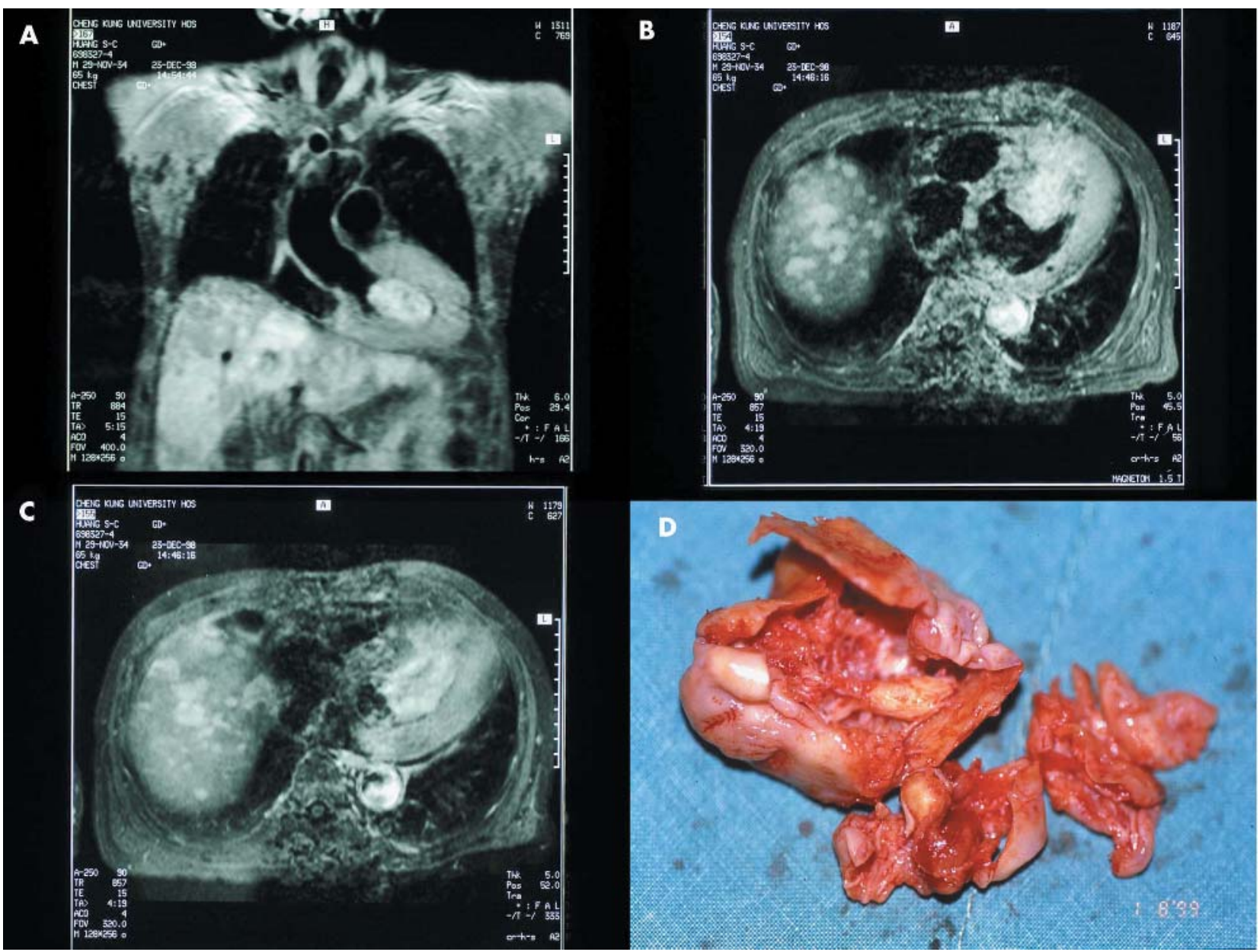

Figure 1 (A, B, C) Sagittal and axial T1 weighted magnetic resonance imaging with fat saturation after gadolinium enhancement showing a well enhanced tumour within the left ventricle, distinct from the myocardium, and about $6 \mathrm{~cm}$ in size. Multiple enhanced tumours of various sizes were also found throughout the liver. Signal voids were noted in the left ventricle and right lobe of the liver, consistent with calcification. (D) The excised gross tumour.

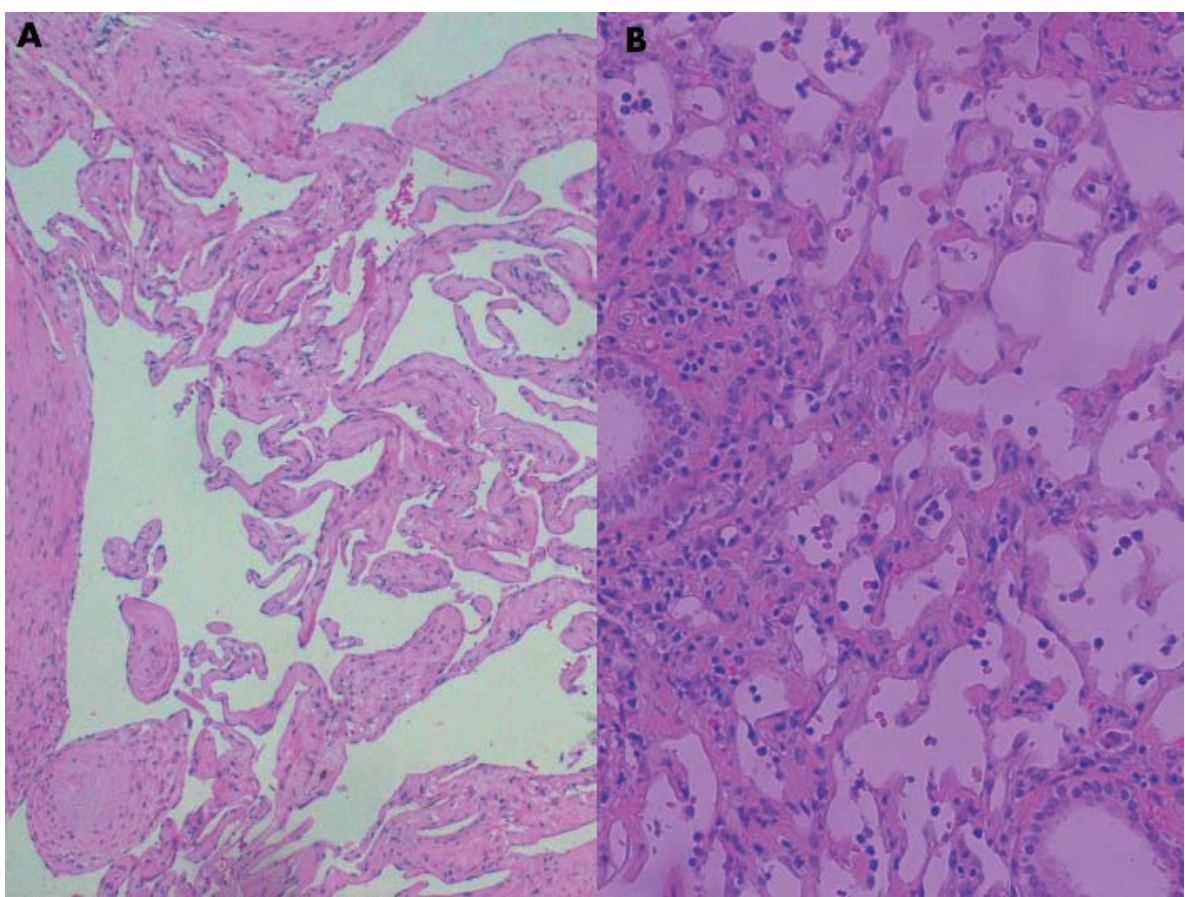

Figure 2 (A) Section of material resected from the cardiac mass; numerous papillae are seen, covered by a single layer of innocent looking endothelial cells (haematoxylin and eosin; original magnification $\times 40$ ). (B) Section of material resected from hepatic nodules, showing the same papillary structure as in the cardiac mass, with sparing of the portal areas. (Haematoxylin and eosin; original magnification $\times 100$ ) 
albeit puzzling, initial diagnosis. Despite the apparently well progressed nature of the disease, complete surgical resection still seemed indicated, owing to the potential for the floating mass in the left ventricular cavity to induce ventricular arrhythmias, sudden death, or systemic dissemination of the thrombus. Ultimately this led to the correct diagnosis. This case serves to highlight how hypervascular cardiac or hepatic tumours may in fact be of vascular origin, rather than metabolically active malignant or metastatic tumours with correspondingly increased vascularity.

\section{Authors' affiliations}

C-D Kan*, Y-J Yang, Department of Surgery, National Cheng Kung

University Hospital, Tainan, Taiwan

C-T Yae, Department of Pathology, National Cheng Kung University Hospital

*Also Institute of Clinical Medicine, Medical College, National Cheng Kung University
Correspondence to: Dr Y-J Yang, Division of Cardiovascular Surgery, Department of Surgery, National Cheng Kung University Hospital, 138 Sheng-Li Road, Tainan 704, Taiwan; kcd56@mail.ncku.edu.tw

Accepted 22 April 2004

\section{REFERENCES}

1 Melato M. Costantinides F, Pandullo C, et al. Masson's pseudoangiosarcoma, alias intravascular papillary endothelial hyperplasia, in dissecting aortic

aneurysm caused by cystic medionecrosis. Cardiovasc Pathol 2001;10:247-9.

2 de Courten A, Kuffer R, Samson J, et al. Intravascular papillary endothelial hyperplasia of the mouth: report of six cases and literature review. Oral Dis 1999:5:175-8.

3 Tabry IF, Nassar VH, Rizk G, et al. Cavernous hemangioma of the heart: case report and review of the literature. J Thorac Cardiovasc Surg 1975;69:415-20.

4 Abad C, Campo E, Estruch R, et al. Cardiac hemangioma with papillary endothelial hyperplasia: report of a resected case and review of the literature. Ann Thorac Surg 1990;49:305-8.

5 Crilley JG, Ritchie C, de Belder MA. Papillary endothelial hyperplasia in a TEC coronary atherectomy specimen. Heart 1997;77:572-3.

6 Kim HS, Park EJ, Lee JH, et al. Intravascular papillary endothelial hyperplasia in the kidney of a child. Virchows Arch 2000;436:398-400. 